\title{
Can Neural Image Captioning be Controlled via Forced Attention?
}

\author{
Philipp Sadler, Tatjana Scheffler and David Schlangen \\ Department of Linguistics \\ Research Focus Cognitive Sciences \\ University of Potsdam, Germany \\ \{name.surname\}@uni-potsdam.de
}

\begin{abstract}
Learned dynamic weighting of the conditioning signal (attention) has been shown to improve neural language generation in a variety of settings. The weights applied when generating a particular output sequence have also been viewed as providing a potentially explanatory insight into the internal workings of the generator. In this paper, we reverse the direction of this connection and ask whether through the control of the attention of the model we can control its output. Specifically, we take a standard neural image captioning model that uses attention, and fix the attention to predetermined areas in the image. We evaluate whether the resulting output is more likely to mention the class of the object in that area than the normally generated caption. We introduce three effective methods to control the attention and find that these are producing expected results in up to $27.43 \%$ of the cases.
\end{abstract}

\section{Introduction}

Sequential deep learning language models with an attention mechanism are able to use not only the immediately previous inputs, but can involve context by "attending to" select parts of the whole input sequence at each time step. This has first been shown to be helpful for neural machine translation, which operates on sequences of words. Here, deep learning networks with attention are capable to jointly learn the alignment and translation of languages (Bahdanau et al., 2014; Luong et al., 2015).

From the beginning, the dynamics of the attention while generating output sequences has been seen as providing insight into the workings of the models, if only qualitatively. This is in particular applicable to the interdisciplinary fields of natural language processing and computer vision like image captioning. For example, Xu et al. (2015)

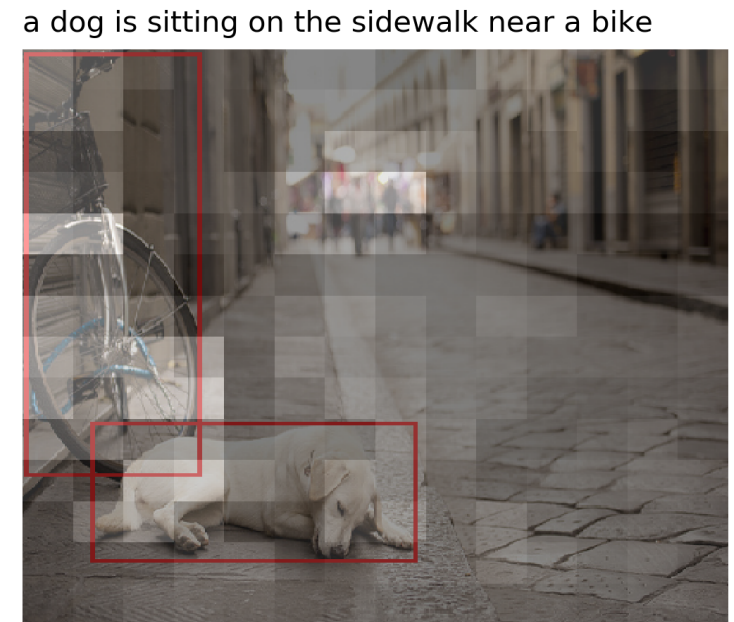

Figure 1: A caption generated by an image captioning model. The attention is pixelated and summed up over all time steps. In addition, the dog and the bicycle are framed with the corresponding bounding boxes.

overlay the attention weights over the input image and show how it shifts while generating the image caption step by step. We show a variant of this visualisation type in Figure 1. The implicit argument at least seems to be that this is informative, because there is a causal relation between where the attention is placed and what is being produced, which has been critically discussed recently (Serrano and Smith, 2019; Jain and Wallace, 2019).

In this paper, we address the question of whether this assumed connection can be used to assert additional control over the generation process. We train a caption generation model with spatial attention in the usual way, but then at test time override its attention mechanism and force it to attend to pre-determined parts of the image. Does this cause the generated output to be different from what would otherwise have been produced, in predictable ways? Our results show that this expectation is partially supported. 


\section{Related Work}

There have been several attempts to achieve more control over neural language generation. Anderson et al. (2017) control the output of a captioning model at test time with an enhanced beam search. An external system is generating image tags as a control signal at the decoder level. They show that adding the additional hints for the generation process actually improves the performance for out-of-domain captioning. Although this approach works, there is no attention effecting mechanism involved. Zarrieß and Schlangen (2018) evaluate a "trainable decoding" approach that inserts taskspecific concerns into the decoding process.

Interfering with the attention mechanism after training, however, has to our knowledge been tried less often. Cornia et al. (2018) train a captioning model not only to learn the distribution for images and sentences, but also for bounding boxes and noun chunks. In addition, the model has to learn when to switch between boxes. As a result, the captioning model is controllable by a bounding box sequence provided as an input to the network at test time. Although this approach has been shown to work well, here the model is explicitly designed to be controllable.

In contrast to the previous approaches, we assume that an attention-aware captioning model is inherently controllable by its spatial attention. In such a sense, our approach is an inverse of the visual grounding task. In one of their experiments, Rohrbach et al. (2016) try to localize phrases within an image by deriving bounding boxes from the spatial attention of a specially trained model. We try to reverse this direction and fix the attention to manually chosen parts of the image after training to generate captions about that region.

\section{An Attentive Image Captioning Model}

We implement a standard neural image captioning architecture that uses spatial attention, which has shown to correlate objects within the image with spatial attention (Xu et al., 2015). As a modification, we use the image features of the pooling layer in the fifth convolutional block like Yang et al. (2015). This modification lead to higher BLEU scores for our setup.

The network is trained on the training split of the MSCOCO dataset for the Captioning Challenge 2015. This split provides five ground-truth captions for each of the 82,783 images. The dataset images are resized to $448 \times 448$ pixels not keeping the aspect ratio. We apply only basic tokenization on the captions. Then the captions of the dataset are prepared to contain only captions that have a maximal length of 16 words. Furthermore, the vocabulary is constrained to the 10,000 most common words and we discard captions that are containing words not included in this vocabulary. The caption-image pairs are shuffled randomly before training. As in the work of Xu et al. (2015), we apply dropout and use the Adam optimizer to minimize the loss function

$$
L=-\log (P(y \mid x))+\lambda \sum_{i}^{L}\left(1-\sum_{t}^{C} \alpha_{i t}\right)^{2}
$$

with the alpha-regularizer $\lambda, L$ as the number of image features, $C$ as the caption length and $\alpha_{i t}$ as the spatial attention for an image feature at a specific time-step. The alpha-regularizer $\lambda$ constraints the caption generator to distribute the spatial attention more equally among the image areas during the whole generation process. $\mathrm{Xu}$ et al. (2015) noted that this regularizer is important for the resulting overall BLEU score, but they did not mention the exact value to be chosen. Therefore we tried $0.001,0.005,0.010$ and found that $\lambda=0.005$ produces the best scores.

Our best model achieves 69.8, 51.8, 37.2, 26.6 in BLEU-1,2,3,4, respectively. Thus we were able to partially increase on the reported scores on the same validation split. Xu et al. (2015) reported $70.7,49.2,34.4,24.3$, so our model is worse by -0.9 in BLEU-1 scores, but better by $+2.6,+2.8$, +2.3 in the other scores.

\section{Methods}

Assuming that a sufficiently well trained captioning system is capable of talking about a variety of objects and object configurations, we expect that the caption generation process is controllable when forcing the attention to specific parts in the image. We create a dataset for this specific captioning task using the MSCOCO validation split for the Detection Challenge 2015, which provides one or more bounding boxes for each of the 40,504 images (Lin et al., 2014). The boxes frame distinct (but possibly overlapping) objects in the images that are each labelled with one of 80 categories. 


\subsection{Constructing Spatial Attention Vectors}

The distinct objects in the images are framed with bounding boxes which are rectangles defined by their width, height and the xy-coordinates of the left upper corner. We discard all bounding boxes that are smaller than the median size, because the model less likely attends to small objects in the images. From the remaining boxes we derive the spatial attention vectors for the experiments.

To construct a spatial attention vector, we define a matrix $Z \in \mathbb{Z}^{W \times H}$ with $W \times H$ as the according image size and a box coordinate space $B \in \mathbb{Z}^{2}$ with each point that falls into the box. Given these we set the values in the matrix as the following:

$$
\alpha_{x y}=\left\{\begin{array}{l}
255 \text { if }(x, y) \in B \\
0 \text { otherwise }
\end{array}\right.
$$

We use 255 as the attention values to align to RGB format, so that we can easily present the maps along with the their images. We resize the matrix to $14 \times 14$ using nearest neighbor downsampling not keeping the aspect ratio. Finally, the matrix is flattened to a 196-dimensional vector and the softmax function is applied, so that $\sum \alpha_{i}=1$ and $\alpha \in[0,1]$ is guaranteed like in the implementation of Xu et al. (2015). An important detail is that no value is actually zero. The model is still allowed to include image aspects outside the boxes for the caption generation. In addition, when using 255 as an initial attention value, we found that we need to interpolate for each vector the pixels values following $[0,255] \rightarrow[0,1]$, because otherwise the softmax results in too much weight on individual spatial areas and leads to qualitative worse captions e.g. the model is referring to polar bear for the dog on the ground.

\subsection{Forcing the Spatial Attention}

The trained image captioning model has to produce what we call a box caption for each constructed attention vector. That is, the spatial attention is derived from the bounding box like in section 4.1 and applied to the model in one of the following ways.

(a) Unlimited step-wise fixed attention. For this experiment, we feed the spatial attention vector at each time step to the model for the whole caption generation process. The model's own predicted attention is dismissed.

\begin{tabular}{|l|c|c|}
\hline & \multicolumn{2}{|c|}{ Sensitivity } \\
& general (diff) & method (diff) \\
\hline unlimited & $88.68(0.55)$ & $\mathbf{5 2 . 6 5}(0.54)$ \\
\hline limited-3 & $85.23(0.55)$ & $35.20(0.55)$ \\
limited-6 & $87.90(0.56)$ & $46.49(0.55)$ \\
limited-9 & $\mathbf{8 8 . 8 8}(0.55)$ & $51.81(0.54)$ \\
\hline additive-1 & $85.51(0.54)$ & $33.43(0.51)$ \\
additive-2 & $87.26(0.54)$ & $41.25(0.53)$ \\
additive-3 & $85.49(0.54)$ & $44.29(0.52)$ \\
\hline
\end{tabular}

Table 1: The degree of sensitivity as the percentage of 117,798 box captions which deviate from the control (method sens.) or self-attending (general sens.) caption in at least a single word. The differentness for the according subset is given in parentheses as WER scores.

(b) Limited step-wise fixed attention. We feed the spatial attention vector for the first $i=$ $\{3,6,9\}$ time steps which are empirically chosen. After the $t_{i}$ time step, the model is again "free to choose" the spatial attention depending on its state and the previous word.

(c) Step-wise additive attention. At each time step, the spatial attention vector is added to the one predicted by the model. We introduce a factor to control the weight of the externally induced attention and divide by the according term plus 1 .

\section{Results}

For the test image shown in Figure 1, the model produces the caption "a dog is laying down on the street" with unlimited fixed attention on the dog, whereas when forcing the attention on the bicycle: "a bicycle parked in front of a bicycle".

Likewise, when forcing the attention on the bike, the model is producing bike related captions "a bicycle parked in front of a building" for our limited step-wise configurations. In contrast to that, additive attention in this case leads to dog related captions "a dog is sitting on the sidewalk next to a bike" for lower weights up to two and bike related captions "a bicycle parked in front of a building" for weights higher than two. More examples can be found in the supplementary material.

\subsection{Quantitative Analysis: Sensitivity}

The qualitative results show that the model is capable to react to changes in its spatial attention. As a measurement for this capability, we suggest the degree of sensitivity. Here, we quantify how 


\begin{tabular}{|l|c|c|c|c|}
\hline & \multicolumn{3}{|c}{ Controllable } & \multicolumn{2}{c|}{ and distinct } \\
& k @ 1 & k @ 5 & k @ 1 & k @5 \\
\hline unlimited & $\mathbf{2 8 . 5 6}$ & $\mathbf{5 8 . 1 7}$ & $\mathbf{9 . 0 0}$ & $\mathbf{2 1 . 3 9}$ \\
\hline limited-3 & 26.36 & 50.84 & 6.89 & 15.24 \\
limited-6 & 27.69 & 52.75 & 8.21 & 17.86 \\
limited-9 & 27.32 & 52.94 & 7.85 & 18.03 \\
\hline additive-1 & 25.86 & 52.89 & 6.27 & 17.23 \\
additive-2 & 26.98 & 52.28 & 7.26 & 16.70 \\
additive-3 & 27.35 & 53.83 & 7.33 & 18.69 \\
\hline
\end{tabular}

Table 2: The degree of controllability as the percentage of box captions containing their category in relation to all 117,798 box captions. The degree for the distinct share is based on 87,033 (k@1) or 58,407 (k@5) box captions with before unmentioned categories.

often the resulting box captions deviate from the normally generated caption for an image. In the following, we call the normally generated caption a self-attending caption, because the attention is "freely chosen" by the model.

As shown in Table 1, the model has the highest general sensitivity for the limited-9 fixed attention method where $88.88 \%$ of the box captions differ from the self-attending caption in at least a single word. We also compute the WER scores for these subsets and see that on average the box captions are changed in every second word in comparison to the self-attending caption.

In addition, we indicate whether the model's changes in caption generation are related to specific attention forcing methods or a method unrelated phenomenon. To do so, we let the model produce a control caption where the fixed spatial attention has been distributed uniformly over the whole image. This makes it possible to study the effect of the individual forcing methods.

The highest degree of method specific sensitivity is measured for the unlimited fixed attention method as depicted in Table 1. Here, 52.65\% of the box captions differ to the control caption in at least one word. This indicates that among the presented methods, the unlimited spatial fixation is the most effective attention induction method.

\subsection{Quantitative Analysis: Controllability}

Finally, we expect that the produced box captions are referring to objects in the bounding boxes from which the spatial attention vectors are constructed. Thus we evaluate the degree of controllability of our forcing methods by checking that the box cap- tions include the according box categories ( $\mathrm{k} @ 1) .{ }^{1}$

Table 2 shows the highest degree of controllability for the unlimited configuration, which results in $28.56 \%$ of the cases in a box caption that includes its according box category. As the set of $\mathrm{COCO}$ categories is rather restrictive, and e.g. only states "person" where a caption might say "woman" or "man", we also check for the five nearest neighbors in cosine distance of the model's learned word embedding space (k@5). Still, the limited- 6 configuration results in the highest score with $55.58 \%$, in which, for example, the box caption contains the category name "person" or one of its neighbors ("man", "woman", "guy" or "girl").

Furthermore, we compute the degree of controllability within the more interesting distinct subset. In cases where the model already refers to objects within the bounding box, because they include the main objects of an image, we cannot conclude whether the forcing methods have a controllable impact on the resulting captions. Thus, for the distinct subset we discard bounding boxes from the evaluation, which have categories attached that are already included in the standardly produced caption (nearest neighbors accordingly).

Table 2 shows for the distinct subset that in $9.00 \%$ of the cases the resulting caption includes the box category, when the spatial attention is focusing on something new (not mentioned before) in the image using the unlimited fixed attention method. The unlimited configuration has also the highest degree with $21.39 \%$, when we also allow the five nearest neighbors to be included.

\section{Discussion and further work}

The results show that a caption generation model with spatial attention is controllable by the presented forcing methods. The forced model produces predictable results in up to $28.56 \%$ of the cases. These results provide evidence that the model is inherently learning to react to changes in the spatial attention, although the learning task is a more general one. Therefore these results show that specific types of attention like spatial attention might be useful control mechanisms.

The evaluation is difficult, because we use a general purpose dataset in MSCOCO. For example, the most common category in the dataset is

\footnotetext{
${ }^{1}$ The defined metric provides a lower boundary on the performance of attention control, since we compare freely generated captions with a restricted list of classes. We leave a manual evaluation for future work.
} 
"person", which is also the most diverse one. We tried to tackle this problem by also looking for the nearest neighbors of the categories and achieved up to $21.39 \%$ matches in the relevant subset.

Future work will include building and using cleaner and more balanced datasets for the proposed evaluation task. The model's performance is expected to improve when trained on a larger dataset like Visual Genome (Krishna et al., 2016). We think that modifying the spatial attention of a standard neural image captioning model introduces an interesting new research direction for natural language generation, which will allow researchers to handle and understand the complexities of these models more easily.

\section{References}

Peter Anderson, Basura Fernando, Mark Johnson, and Stephen Gould. 2017. Guided Open Vocabulary Image Captioning with Constrained Beam Search. In Proceedings of the 2017 Conference on Empirical Methods in Natural Language Processing, pages 936-945, Copenhagen, Denmark. Association for Computational Linguistics.

Dzmitry Bahdanau, Kyunghyun Cho, and Yoshua Bengio. 2014. Neural Machine Translation by Jointly Learning to Align and Translate. arXiv:1409.0473 [cs, stat]. ArXiv: 1409.0473.

Marcella Cornia, Lorenzo Baraldi, and Rita Cucchiara. 2018. Show, Control and Tell: A Framework for Generating Controllable and Grounded Captions. arXiv:1811.10652 [cs]. ArXiv: 1811.10652.

Sarthak Jain and Byron C. Wallace. 2019. Attention is not Explanation. arXiv:1902.10186 [cs]. ArXiv: 1902.10186.

Ranjay Krishna, Yuke Zhu, Oliver Groth, Justin Johnson, Kenji Hata, Joshua Kravitz, Stephanie Chen, Yannis Kalantidis, Li-Jia Li, David A. Shamma, Michael S. Bernstein, and Fei-Fei Li. 2016. Visual Genome: Connecting Language and Vision Using Crowdsourced Dense Image Annotations. arXiv:1602.07332 [cs]. ArXiv: 1602.07332.

Tsung-Yi Lin, Michael Maire, Serge Belongie, Lubomir Bourdev, Ross Girshick, James Hays, Pietro Perona, Deva Ramanan, C. Lawrence Zitnick, and Piotr Dollár. 2014. Microsoft COCO: Common Objects in Context. arXiv:1405.0312 [cs]. ArXiv: 1405.0312 .

Jiasen Lu, Jianwei Yang, Dhruv Batra, and Devi Parikh. 2016. Hierarchical Question-image Coattention for Visual Question Answering. In Proceedings of the 30th International Conference on Neural Information Processing Systems, NIPS'16, pages 289-297, USA. Curran Associates Inc. Event-place: Barcelona, Spain.

Thang Luong, Hieu Pham, and Christopher D. Manning. 2015. Effective Approaches to Attentionbased Neural Machine Translation. In Proceedings of the 2015 Conference on Empirical Methods in Natural Language Processing, pages 14121421, Lisbon, Portugal. Association for Computational Linguistics.

Anna Rohrbach, Marcus Rohrbach, Ronghang $\mathrm{Hu}$, Trevor Darrell, and Bernt Schiele. 2016. Grounding of Textual Phrases in Images by Reconstruction. arXiv:1511.03745 [cs], 9905:817-834. ArXiv: 1511.03745 .

Sofia Serrano and Noah A. Smith. 2019. Is Attention Interpretable? arXiv:1906.03731 [cs]. ArXiv: 1906.03731.

Kelvin $\mathrm{Xu}$, Jimmy Lei Ba, Ryan Kiros, Kyunghyun Cho, Aaron Courville, Ruslan Salakhutdinov, Richard S. Zemel, and Yoshua Bengio. 2015. Show, Attend and Tell: Neural Image Caption Generation with Visual Attention. In Proceedings of the $32 \mathrm{Nd}$ International Conference on International Conference on Machine Learning - Volume 37, ICML'15, pages 2048-2057, Lille, France. JMLR.org.

Zichao Yang, Xiaodong He, Jianfeng Gao, Li Deng, and Alex Smola. 2015. Stacked Attention Networks for Image Question Answering. arXiv:1511.02274 [cs]. ArXiv: 1511.02274.

Sina Zarrieß and David Schlangen. 2018. Decoding Strategies for Neural Referring Expression Generation. In Proceedings of the International Conference on Natural Language Generation (INLG). 